Breaking axi-symmetry in stenotic flow lowers the critical transition Reynolds number

J. Samuelsson, , O. Tammisola, , and M. P. Juniper

Citation: Phys. Fluids 27, 104103 (2015); doi: 10.1063/1.4934530

View online: http://dx.doi.org/10.1063/1.4934530

View Table of Contents: http://aip.scitation.org/toc/phf/27/10

Published by the American Institute of Physics 


\title{
Breaking axi-symmetry in stenotic flow lowers the critical transition Reynolds number
}

\author{
J. Samuelsson, ${ }^{1, a)}$ O. Tammisola, ${ }^{2, b)}$ and M. P. Juniper ${ }^{3}$ \\ ${ }^{1}$ Harvard-MIT Division of Health Sciences and Technology, MIT, Cambridge, \\ Massachusetts 02139, USA \\ ${ }^{2}$ Faculty of Engineering, The University of Nottingham, University Park, \\ Nottingham NG72RD, United Kingdom \\ ${ }^{3}$ Department of Engineering, University of Cambridge, Trumpington Street, \\ Cambridge CB2 1PZ, United Kingdom
}

(Received 26 February 2015; accepted 21 September 2015; published online 27 October 2015)

\begin{abstract}
Flow through a sinuous stenosis with varying degrees of non-axisymmetric shape variations and at Reynolds number ranging from 250 to 750 is investigated using direct numerical simulation (DNS) and global linear stability analysis. At low Reynolds numbers $(R e<390)$, the flow is always steady and symmetric for an axisymmetric geometry. Two steady state solutions are obtained when the Reynolds number is increased: a symmetric steady state and an eccentric, non-axisymmetric steady state. Either one can be obtained in the DNS depending on the initial condition. A linear global stability analysis around the symmetric and non-axisymmetric steady state reveals that both flows are linearly stable for the same Reynolds number, showing that the first bifurcation from symmetry to antisymmetry is subcritical. When the Reynolds number is increased further, the symmetric state becomes linearly unstable to an eigenmode, which drives the flow towards the non-axisymmetric state. The symmetric state remains steady up to $R e=713$, while the non-axisymmetric state displays regimes of periodic oscillations for $R e \geq 417$ and intermittency for $R e \gtrsim 525$. Further, an offset of the stenosis throat is introduced through the eccentricity parameter $E$. When eccentricity is increased from zero to only $0.3 \%$ of the pipe diameter, the bifurcation Reynolds number decreases by more than $50 \%$, showing that it is highly sensitive to non-axisymmetric shape variations. Based on the resulting bifurcation map and its dependency on $E$, we resolve the discrepancies between previous experimental and computational studies. We also present excellent agreement between our numerical results and previous experimental results. (C) 2015 AIP Publishing LLC. [http://dx.doi.org/10.1063/1.4934530]
\end{abstract}

\section{INTRODUCTION}

Arteriosclerosis is the narrowing of arteries caused by buildup of plaque along the inner artery wall. ${ }^{1}$ This narrowing and re-expansion of the artery wall is known generally as a stenosis and can be modelled as a converging-diverging pipe. A stenosis predisposes the artery to thrombosis (blood clotting) and eventually, when the blood clot detaches from the artery wall, to infarction. ${ }^{2}$ This often causes a heart attack, a stroke, or other tissue damage. ${ }^{2}$

It is important to know whether or not a stenosis is likely to cause thrombosis. This is because surgical intervention itself can cause thrombosis and be more dangerous than the stenosis it is intended to cure. ${ }^{3}$ The medical community, therefore, would like to be able to categorize stenoses into those that are likely to cause thrombosis and those that are not. This will only be possible with a rigorous understanding of the flow within a stenosis. ${ }^{4}$ For example, low and oscillatory wall

\footnotetext{
a) Previously at KTH Mechanics, KTH Royal Institute of Technology, Osquars Backe 18, SE-10044 Stockholm, Sweden.

b) Also at KTH Mechanics, KTH Royal Institute of Technology, Osquars Backe 18, SE-10044 Stockholm, Sweden.
} 
shear stress plays a key role in plaque formation on artery walls ${ }^{5}$ and therefore in the triggering and growth of stenoses. These shear stresses arise as a direct consequence of the flow within the stenosis, in particular its stability, which is the subject of this paper.

Previous studies of stenotic flows have reported steady flow at low Reynolds numbers and transition to unsteady flow as the Reynolds number increases. The reported critical transition Reynolds number varies greatly, however, between different studies. Ahmed and Giddens ${ }^{6}$ conducted an experimental investigation of an axisymmetric stenosis in which the radius varied sinusoidally over a length of two pipe diameters, with blockage ratio from $25 \%$ to $75 \%$ and $R e=500$ and 1000 . At $75 \%$ blockage ratio, non-stationary flow was first observed at $R e=500$. Vétel $e t a{ }^{7}{ }^{7}$ used a slightly different stenosis model in the shape of two intersecting circular arcs, also with two pipe diameters length and $75 \%$ blockage ratio. The Reynolds number ranged from 116 to 1160 , and non-stationary flow was observed for $R e>400$. Griffith et al. ${ }^{8}$ did a joint numerical-experimental study of the effect of blockage ratio, from $20 \%$ to $95 \%$, and Reynolds numbers from 50 to 2500 using a stenosis model with a semi-circular axial profile. Experimental results showed non-stationary flow at $R e \approx 400$ for a stenosis blockage ratio of $75 \%$. Sherwin and Blackburn ${ }^{9}$ performed a numerical study implementing the same geometry as that used by Ahmed and Giddens ${ }^{6}$ at a blockage ratio of $75 \%$, using direct numerical simulation (DNS) and global linear stability analysis in the Reynolds number regime $400 \leq R e \leq 800$. They showed that the flow is stationary, symmetric, and stable up to a critical Reynolds number of $R e_{c}=722$, at which point the symmetric flow becomes unstable. Turbulent flow was observed for $R e>688$ in a secondary solution branch where the jet was skewed through a Coanda-type wall attachment. ${ }^{10}$ Varghese, Frankel, and Fischer ${ }^{11}$ performed DNS-simulations for $R e=500,1000$ and $E=0,5 \%$. Non-stationary flow was only observed at $R e=1000$ and $E=5 \%$. Table I gives an overview of previous studies and their investigated regions in $(R e, E)$-space along with numerical implementation parameters.

The reported flow dynamics differ in these studies. According to experimental results by Vétel et al., ${ }^{7}$ the flow is nearly symmetric for $R e \leq 302$, but by $R e=348$, the flow has undergone a Coanda-type wall attachment to one side and is strongly skewed. Varghese, Frankel, and Fischer, ${ }^{11}$ on the contrary, reported a symmetric jet for $E=0$ at $R e=500$ and 1000 , the jet being skewed only at $E=5 \%$ and $R e=500$. For $R e>440$, Vétel et al. ${ }^{7}$ reported intermittent flow with alternating turbulent and laminar phases. As the Reynolds number increased, the turbulent phases became longer in time and the post-stenotic turbulent region moved further upstream until turbulent phases took over at $R e \approx 922$ and intermittency was gone. This intermittency pattern was also reported by Sherwin and Blackburn, ${ }^{9}$ but at higher Reynolds numbers, $R e>580$. The non-stationary flow in Ahmed and Giddens ${ }^{6}$ was reported as discrete frequency oscillations at $R e=500$ and full turbulence was observed in the post-stenotic region at $R e=1000$. Griffith et al. ${ }^{8}$ observed Kevin-Helmholtz waves in the post-stenotic recirculation region, growing in amplitude while being convected downstream, thus demonstrating convective instability.

TABLE I. Overview of previous studies' investigated regions in $(R e, E)$ space, projected at a blockage ratio of $75 \%$, with numerical implementation parameters. Studies in italics are experimental. The mesh resolution is given in approximate number of nodes. Sherwin and Blackburn ${ }^{9}$ did a 2D study, hence, the relatively low number of gridpoints.

\begin{tabular}{|c|c|c|c|c|}
\hline Study & $\operatorname{Re}$ & $\mathrm{E}(\%)$ & $\begin{array}{c}\text { Mesh } \\
\text { resolution }\end{array}$ & $\begin{array}{c}\text { Domain } \\
\text { size }\end{array}$ \\
\hline Vétel et al. ${ }^{7}$ & $116-1160$ & 0 & & \\
\hline Ahmed and Giddens ${ }^{6}$ & 500,1000 & 0 & & \\
\hline Griffith et al. ${ }^{8}$ & $50-2500$ & 0 & N/A & $81 \mathrm{D}$ \\
\hline Sherwin and Blackburn ${ }^{9}$ & $400-800$ & 0 & 38000 & $50 \mathrm{D}$ \\
\hline Griffith et al. ${ }^{12}$ & $1-400$ & $0-5$ & 200000 & $31 \mathrm{D}$ \\
\hline Varghese, Frankel, and Fischer ${ }^{11}$ & 500,1000 & 0,5 & 1200000 & N/A \\
\hline Present study & $250-750$ & $0-5$ & 3646080 & $31 \mathrm{D}$ \\
\hline
\end{tabular}


Griffith et al. ${ }^{12}$ performed a numerical DNS study and showed that the discrepancy in the steady flow regime between the experimental results reported by Vétel et al. ${ }^{7}$ and the numerical investigations can be accredited to non-axisymmetric changes in the stenosis' shape, inherent in all experimental systems. However, the investigated Reynolds number ranged from $R e=1$ to 400 and thus did not cover the non-stationary flow regime, nor was any stability analysis performed. Sherwin and Blackburn ${ }^{9}$ did notice a subcritical bifurcation, but the study was limited to a perfectly axisymmetric geometry. Thus, the hydrodynamic stability's sensitivity to non-axisymmetric shape variations remains unexplored. This paper aims to bridge that gap and to quantify the effect of non-axisymmetric shape variations to see whether or not this is the source of discrepancy in numerics and experiments, in the stationary as well as the non-stationary regime. To accomplish this, DNS and global linear stability analysis are used. Modal stability analyses for a range of different eccentricities are performed, to see whether the leading eigenmode becomes unstable at lower Reynolds numbers for non-axisymmetric geometries. If a modal instability is observed at a certain Reynolds number for slightly non-axisymmetric geometries, but not for symmetric ones, this could explain why the critical Reynolds numbers are different in numerical studies (symmetric geometries) and experimental studies. The shape of the bifurcation, i.e., to what degree it is subcritical, is evaluated by varying the initial conditions in the DNS.

\section{METHODS}

\section{A. Problem setup}

The simplified stenosis geometries are adopted from Varghese, Frankel, and Fischer ${ }^{11}$ and shown in Figure 1. The origin of the coordinate system is at the centerline of the throat. The axial coordinate is $z$ and the coordinate in the direction of the eccentricity (labelled the cross-stream coordinate) is $y$. In the following, the star ${ }^{*}$ denotes dimensional coordinates, $\mathbf{u}$ is the velocity vector, and $p$ is the pressure.

The smallest diameter at the throat of the constriction is $0.5 \mathrm{D}$, giving a ratio of 0.25 between the minimum and maximum cross-sectional areas. This blockage ratio of $75 \%$ represents the threshold of clinical significance and visibility using present technology. ${ }^{7}$ The radius can be written as

$$
r^{*}\left(z^{*}\right)=0.5 D\left[1-0.25\left(1+\cos \left(2 \pi z^{*} / L\right)\right)\right]
$$

throughout the stenosis, i.e., for $-D<z^{*}<D$. The shape asymmetry consists of an offset of the stenosis walls in the $y$-direction, known as eccentricity. The eccentricity has a sinuous axial profile, defined as

$$
E_{z}\left(z^{*}\right)=0.5 E D\left[1+\cos \left(2 \pi z^{*} / L\right)\right]
$$

so that the eccentricity is zero at the boundaries of the stenosis, $\left|z^{*}\right|=D$, and reaches its peak at the throat where it equals $E$. The wall coordinates in the eccentric stenosis model are

$$
y^{*}=r^{*} \cos (\phi)+E_{z}, \quad x^{*}=r^{*} \sin (\phi), \phi: 0 \rightarrow 2 \pi .
$$

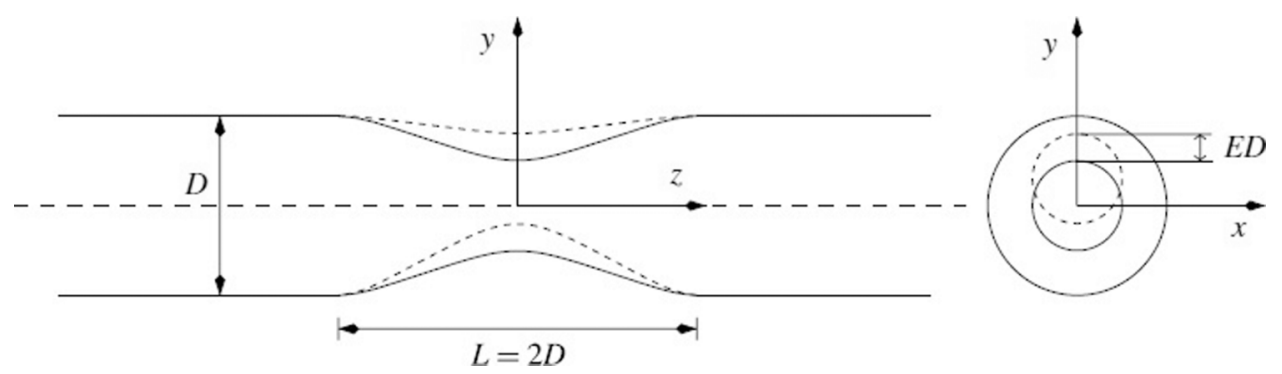

FIG. 1. Schematic of the stenosis geometry under investigation, adapted from Ref. 12. 
The inlet is placed $6 D$ upstream of the throat, and a Poiseuille inlet velocity profile is imposed: $U^{*}=2 \tilde{U}\left[1-4 r^{* 2} / D^{2}\right]$, where $\tilde{U}$ is the average inlet velocity. In the remainder of this paper, nondimensionalized coordinates will be used; $U=U^{*} / \tilde{U}$ and $(x, y, z)=\left(x^{*}, y^{*}, z^{*}\right) / D, t=t^{*} \tilde{U} / D$. The Reynolds number is thus defined as $R e=\tilde{U} D / v$. The problem is defined by two variables: the eccentricity $E$ and the Reynolds number $R e$. Using the diameter of the common carotid artery, Bharadvaj, Mabon, and Giddens ${ }^{13}$ showed that a representative, time-averaged, Reynolds number for this blood vessel is $\tilde{R} e=380$. It was also noted, however, that because blood flow is pulsatile, the peak Reynolds number can be as high as 1200. Most studies on stenotic flows have been in the range of $100<R e<1000$. In this study, the investigated Reynolds number range is from 250 to 750 , and the eccentricity is from $0 \%$ to $5 \%$.

\section{B. Governing equations}

The governing equations for the DNS are the incompressible Navier-Stokes equations with no-slip boundary conditions at the walls and a zero stress boundary condition at the outlet. In global stability analysis, the velocity field (and similarly the pressure) is decomposed as

$$
\mathbf{u}=\mathbf{U}(r, z)+\hat{\mathbf{u}}(r, \phi, z) \exp \sigma t,
$$

where $\mathbf{U}$ is the axisymmetric base flow, and the second term is based on a $3 \mathrm{D}$ global mode ansatz for the disturbance. This leads to the following equation system to be solved:

$$
\begin{gathered}
\sigma \hat{\mathbf{u}}+\hat{\mathbf{u}} \cdot \nabla \mathbf{U}_{b}+\mathbf{U}_{b} \cdot \nabla \hat{\mathbf{u}}=-\nabla \hat{p}+\frac{1}{R e} \nabla^{2} \hat{\mathbf{u}}, \\
\nabla \cdot \hat{\mathbf{u}}=0,
\end{gathered}
$$

where $(\hat{\mathbf{u}}, \hat{p})$ is the spatial shape of the global mode with the complex eigenvalue $\sigma=\sigma_{r}+\sigma_{i}$. Modes with positive $\sigma_{r}$ are unstable. In our case, the unstable modes have $\sigma_{i}=0$ (zero frequency).

\section{Numerical implementation}

The open-source software Nek5000 ${ }^{14}$ is used in this study for DNS-simulations. A threedimensional mesh consisting of 8000 hexahedral elements is used for $R e<400$. Nodes in each element are distributed according to Gauss-Lobatto-Legendre quadrature in every spatial dimension. The polynomial order is $N=5$ in each element. The mesh consists of 80 two dimensional cells in the $x y$-plane (Figure 2(a)) with 100 elements in the axial direction, creating the 8000 element mesh with 1728000 nodes. The domain extends $6 D$ upstream of the stenosis throat and $25 D$ downstream. Since the domain boundaries are transformed analytically from a straight pipe to the given eccentric shape before the domain is discretized, the geometric precision of the pipe wall is to the order of double precision, i.e., $O\left(10^{-16}\right)$ in non-dimensionalized terms. The smallest investigated eccentricity is $0.02 \%$, thus well above the precision level set by numerical approximation.

(a)

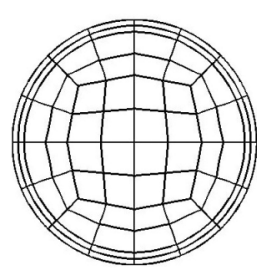

(b)

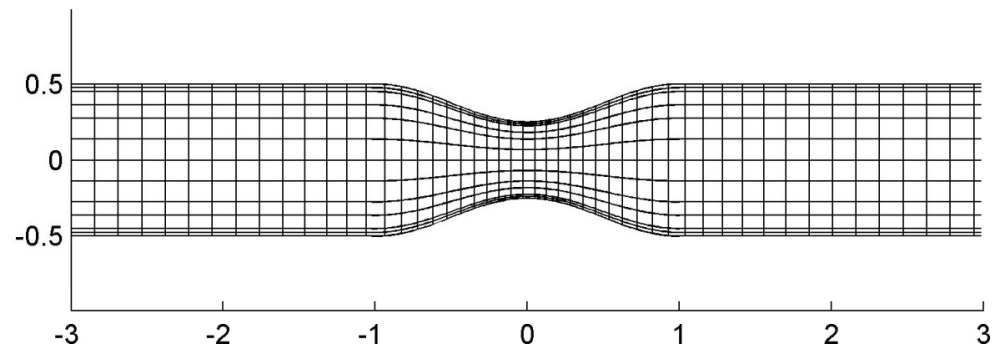

FIG. 2. Spectral element outlines of the computational mesh in the $x y$-plane (a) and in the $z y$-plane $x=0$ (b), zoomed in around the stenosis throat. 
A mesh convergence study has been performed. For $R e=350, E=0.26 \%$, the difference in flow asymmetry $\mu_{y}$ (Equation (5)) is everywhere less than $1.6 \%$ between the 8000 and 16880 element meshes, and $0.4 \%$ between 16880 elements and 33760 elements. The mesh of 8000 elements is therefore concluded to be sufficient for describing the stationary flow at $R e<400$. For $R e>400$, the 8000 element mesh does not produce convergent results with the denser meshes, so the 16880 element mesh of 3646080 nodes is used instead. The 16880 and 33760 element meshes (7 292160 nodes) produce similar results for all cases examined, and all critical Reynolds numbers and eccentricities presented in this paper have been confirmed with the 33760 element mesh. A representative mesh for this study is therefore the 16880 element mesh with 3646080 nodes, which is on display in Figure 2. The 16880 and 33760 meshes contain hexahedral elements of sinusoidally varying length along the axial direction in the region $|z|<2$, such that the element length is two times smaller at $z=0$ than in the region $|z|>2$. A time step of $\Delta t=10^{-3}$ is used for the 8000 and 16880 element meshes in the DNS, and $0.5 \times 10^{-3}$ for the 33670 element mesh. Simulations were performed until the time trace repeated itself, or a steady state was reached in the case of non-oscillatory flow, which usually occurred after 100-400 time units (100 000-400 000 time steps).

\section{Global linear eigenmodes}

The TriGlobal linear eigenmodes are computed directly from ansatz (3). Because the base flows for eccentric stenoses are non-axisymmetric, it is not possible to consider modes with different azimuthal wavenumbers separately. The full three-dimensional eigenpairs of the linearized Navier-Stokes operator are computed using the linearized DNS time-stepper (available in Nek5000) coupled with the implicit restart Arnoldi method implemented in PARPACK. ${ }^{15}$ This matrix-free global stability solver implementation was also used in Tammisola et al. ${ }^{16}$ and was there verified against a FreeFem++ based solver.

\section{Definitions}

For all examined Reynolds numbers, ranging from $R e=250$ to $R e=750$, the flow separates in the diverging section of the stenosis and a jet is formed. The deflection of the jet can be described by tracking flow asymmetry, $\mu_{y}$, as a function of the streamwise coordinate, as was done by Griffith et al. ${ }^{12}$ using the normalized first moment of the streamwise velocity component,

$$
\mu_{y}=\frac{\int y u_{z} d A}{\int u_{z} d A} .
$$

This measure, however, includes the effects of shape asymmetry as well as flow asymmetry. To eliminate the effect of shape asymmetry itself and to be able to compare results for different shapes, we measure the flow asymmetry in the downstream region where the shape is constant using the maximum flow asymmetry $\hat{\mu}_{y}$,

$$
\hat{\mu}_{y}=\max _{z>1}\left\{\mu_{y}(z)\right\}
$$

\section{Validation}

The DNS code was validated against results from Griffith et al. ${ }^{12}$ Griffith et al. ${ }^{12}$ noticed a discontinuity in the asymmetry of the flow when varying eccentricity at around $E \approx 0.25 \%$ and $E \approx 0.18 \%( \pm 0.02 \%)$ for $R e=350$ and $R e=400$, respectively. That same discontinuity was found in this study, occurring at $E \approx 0.27 \%$ and $E \approx 0.17 \%$ for $R e=350$ and $R e=400$, respectively. Maximum flow asymmetry $\hat{\mu}_{y}$ for $R e=350$ and $E=0.1 \%$ was $\hat{\mu}_{y} \approx 0.019$, found at $z \approx 4.3,{ }^{12}$ in agreement with the present study, which finds $\hat{\mu}_{y}=0.0189$ at $z=4.34$. Stability analysis performed around $E=0$ gives the same leading eigenmode as that found by Sherwin and Blackburn. ${ }^{9}$ Further, leading eigenvalues for $E=0$ and $R e=500,600,700$ around the symmetric flow match those presented by Sherwin and Blackburn. ${ }^{9}$ The critical Reynolds number when the symmetric flow turns unstable was found to be $R e_{c}=713$, Sherwin and Blackburn ${ }^{9}$ approximated this value to $R e_{c}=722$ via an interpolation. 
(a)

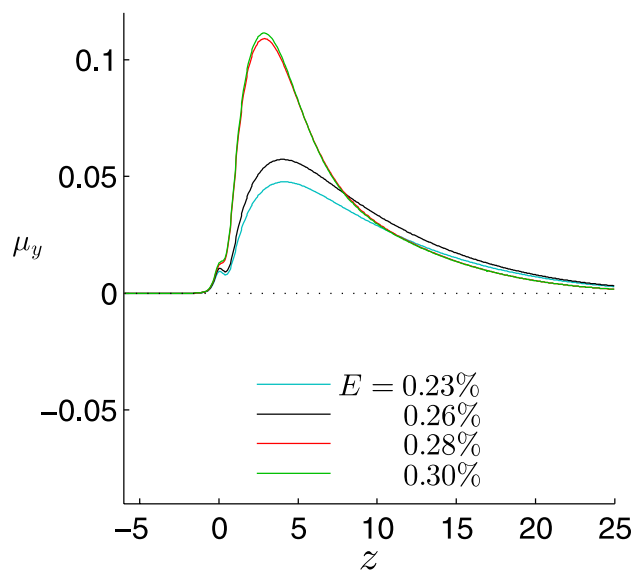

(b)

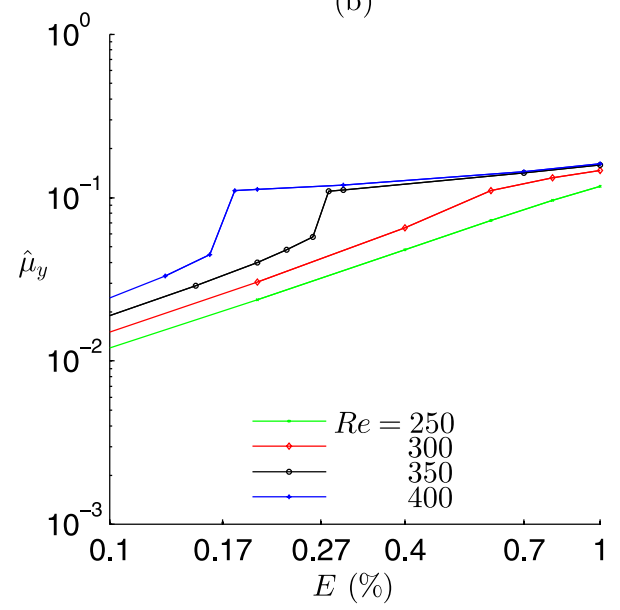

FIG. 3. (a) Flow asymmetry as a function of $z$ for $R e=350$ and $E=0.23 \%, 0.26 \%, 0.28 \%, 0.3 \%$. (b) Maximum flow asymmetry as a function of eccentricity for $R e=250,300,350$, and 400 . All points represent steady, stable solutions found by using a fluid at rest as initial condition.

\section{RESULTS}

\section{A. DNS with a flow at rest as initial condition}

In all cases examined $(250<R e<750)$, the DNS shows that separation occurs in the diverging section of the stenosis, forming a jet in the post-stenotic area (Figure 5(c)). As the jet emerges from the stenosis, it is surrounded by a long, annular recirculation zone. When introducing an eccentricity, the length of the recirculation zone depends on the azimuthal angle $\phi$, the zone being shorter in the upper region of the pipe and longer in the lower region. This is the result of a weak Coanda type wall attachment. ${ }^{10}$ Asymptotic flow states retrieved from the DNS with a flow at rest as initial condition are presented in Figure 3(a) which plots flow asymmetry $\mu_{y}$ against streamwise coordinate $z$ for a range of eccentricities and $R e=350$. Figure 3(a) shows that the maximum flow asymmetry jumps from $\hat{\mu}_{y}=0.06$ to $\hat{\mu}_{y}=0.12$ at around $E_{0}=0.27 \%$. Figure $3(\mathrm{~b})$ plots the maximum flow asymmetry $\hat{\mu}_{y}$ against eccentricity $E$ for different Reynolds numbers.

As Figure 3(b) shows, when eccentricity is increased beyond a certain value $E_{0}$, e.g., $E_{0}=$ $0.27 \%$ for $R e=350$ and $E_{0}=0.17 \%$ for $R e=400$, the flow asymmetry increases abruptly, which was also noted by Griffith et al. ${ }^{12}$ Thus, the flow can be divided into two regimes: branch 1, with the minor flow asymmetry and branch 2 with the greater flow asymmetry. The physics of this new flow regime, branch 2, is largely the same: the flow also exhibits a Coanda type wall attachment to the upper side, but the flow separates further upstream in branch 2 than branch 1, which leads to an increase in flow asymmetry in the post-stenotic region. A difference that will prove significant when increasing the Reynolds number between the two regimes is the shape of the recirculation zone; in branch 2, the lower recirculation zone is considerably thicker, the shear layer is thinner and the flow more susceptible to shear layer instability than branch 1 . This difference will be explained shortly using stability analysis.

\section{B. Stability analysis}

In order to investigate whether the discontinuity observed at $E=E_{0}$ can be attributed to a bifurcation, we perform global linear stability analysis around the flows in branch 1 for various eccentricities. Figure 4(a) shows the real part of the leading eigenvalue $\sigma_{r}$ for different eccentricities at $R e=400$. The analysis shows that the growth rate of the leading eigenvalue increases with eccentricity. Further, the eigenvalue drift seems to be of second order, ${ }^{17}$ demonstrating an application of the theory on second order sensitivity developed by Tammisola et al. ${ }^{16}$ Extrapolating from a quadratic least square fit at $R e=400$ shows that the mode becomes unstable at around $E=0.17 \%$, 
(a)

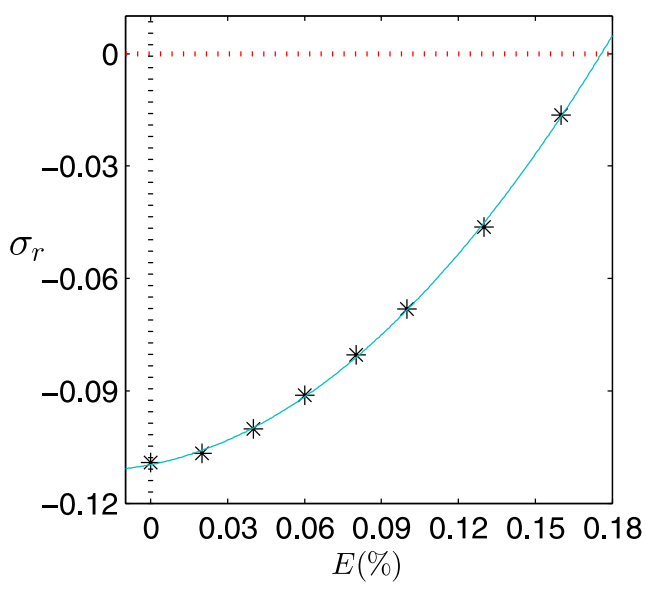

(b)

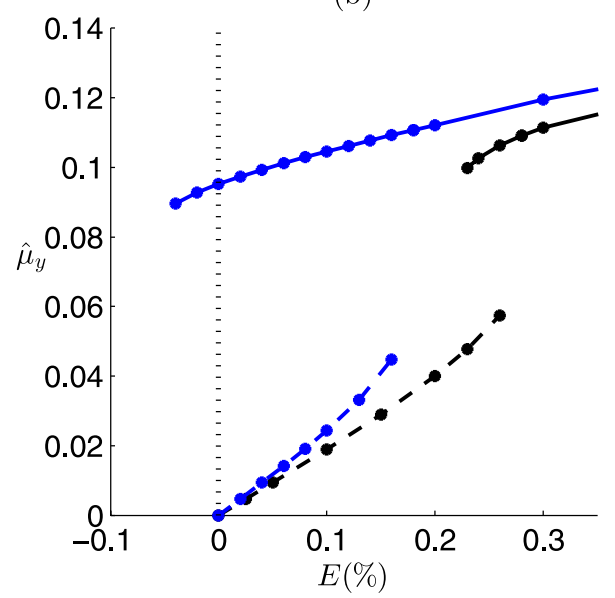

FIG. 4. (a) Growth rate $\sigma_{r}$ of the leading eigenmode in branch 1 for $R e=400$ to eccentricity $E$, along with a quadratic fit. (b) Asymmetry of the steady flows as a function of the eccentricity, $E$, for $R e=350$ (black) and $R e=400$ (blue). For both Reynolds numbers, there is a range of eccentricities in which two steady flows are possible; the dashed lines represent branch 1 and solid lines branch 2.

which matches the discontinuity in Figure 3(b). An identical study performed for $R e=350$ predicts the leading mode to become unstable at $E=0.27 \%$, which is also where the discontinuity occurs in Figure 3(b). It can therefore be concluded that a bifurcation does indeed occur and is responsible for the observed discontinuity.

At $E=0$ (symmetric flow), the leading eigenmode has an azimuthal wavenumber of $m=1$ and is degenerate, with two identical eigenfunctions rotated by $\pi / 2$ in the azimuthal direction relative to each other. These two modes have different shape sensitivities and one of them becomes the sole leading mode when an eccentricity is introduced. The shape of this leading eigenmode is also affected by eccentricity. The azimuthal dependence is $m= \pm 1$ for $E=0$ but is a superposition of different wave numbers, $m=0, \pm 1, \pm 2$ being dominant, for $E>0$. The dominant azimuthal wave numbers of the flow in branch 2 are also $m=0, \pm 1, \pm 2$.

\section{DNS with different flow states as initial conditions}

DNS with different flow states as initial conditions was performed by varying the Reynolds number and eccentricity in the DNS. When varying eccentricity, the initial condition was transformed from the old grid to the new grid (new eccentricity) by pointwise zeroth order extrapolation, i.e., the solution was moved from the old grid to the new grid. This procedure was considered to be accurate, since the changes in eccentricity were typically of the order $O\left(10^{-4}\right)$, which then also was the maximal movement of the grid. Starting from a branch 2 solution, we reduce the eccentricity $E$ and reconverge to branch 2. This allows us to follow branch 2 for $E<E_{0}$. Figure 4(b) shows DNS data for stable, steady solutions that have been retrieved this way for $R e=350$ and $R e=400$. Figure 4(b) reveals a bistable region, e.g., $0.22 \%<E<0.27 \%$ for $R e=350$, in which hysteresis is possible. For $R e=400$, the bistable region extends from the discontinuity observed at $E=0.17 \%$ down to $E=-0.04 \%$. Thus, for $R e=400$, branch 1 is stable for $E<0.17 \%$, which is also shown by Figure 4(a). For $E>0.17 \%$, branch 1 is unstable and branch 2 is stable. For $-0.04 \%<E<$ $0.17 \%$, both branch 1 and branch 2 are stable, hence $-0.04 \%<E<0.17 \%$ constitutes a bistable region for $R e=400$. For $E<-0.04 \%$, branch 2 is unstable and branch 1 is stable. This also shows that there are two stable solutions for the symmetric geometry $E=0$ at $R e=400$. An alternative way of following branch 2 is to vary the Reynolds number and letting eccentricity remain constant. Lowering the Reynolds number in the DNS from $R e=400$ shows that branch 2 vanishes at around $R e=390$ for $E=0$, demonstrating the presence of a bistable region for the axisymmetric stenosis 
for $R e>390$. Figure 5 shows the streamwise velocity of branch 1, branch 2 as well as the leading eigenmode of branch 1, for $R e=400$ and $E=0$, demonstrating the bifurcation.

\section{Influence of different stenosis models}

Some previous studies, e.g., Vétel et al. ${ }^{7}$ and Griffith et al. ${ }^{8}$ used a stenosis model other than the sinusoidally shaped model used in this study. In Vétel et al., ${ }^{7}$ the stenosis was modeled by two intersecting circular arcs. In order to evaluate to what extent the results from the present study can be applied to experimental results by Vétel et al., ${ }^{7}$ DNS simulations for the different stenosis models were performed and compared. Results showed that the flow physics is largely the same and that bifurcation also occurs for this geometry, but for a slightly higher eccentricity. Therefore, when comparing results between these two geometries, one should only expect qualitative agreement in terms of Reynolds number or eccentricity.

\section{E. Bifurcation diagram}

In Figure 5(c), the central jet is deflected in the positive $y$-direction. When the problem is axisymmetric, as it is in Figure 5, the jet can be deflected in any azimuthal direction. Thus, the flow exhibits an axisymmetric subcritical pitchfork bifurcation. Following branch 1 for $E=0$ by increasing the Reynolds number gives steady, symmetric solutions all the way up to the critical Reynolds number $R e_{c}=713$. A bifurcation diagram for $E=0 \%$ and $0.16 \%$ is presented in Figure 6. Following branch 1 for $E=0.27 \%$ by increasing the Reynolds number gives $R e_{c}=350$. Hence, the critical Reynolds number at which the nearly symmetric flow (branch 1) transforms into branch 2 is halved by an asymmetry less than $0.3 \%$ of the pipe diameter, which may well be less than engineering tolerance for pipes. To illustrate the hypersensitivity of branch 1 , consider a pipe with a diameter of $2 \mathrm{~cm}$. When introducing a shape asymmetry to the width of a human hair in this pipe, the critical Reynolds number for transformation to the strongly asymmetric flow of branch 2 decreases by more than $50 \%$.

The bifurcation diagram presented in Figure 6 shows that branch 2 is stable for $\operatorname{Re}>390$ for $E=0$ and $R e>360$ for $E=0.16 \%$. This difference in Reynolds number ( $~ 8 \%)$ is small compared to branch 1, which turns unstable at $R e=713$ for $E=0$ and $R e=400$ for $E=0.16 \%$, constituting a difference in the critical Reynolds number of $\sim 45 \%$. Hence, the stability of branch 1 changes

(a)

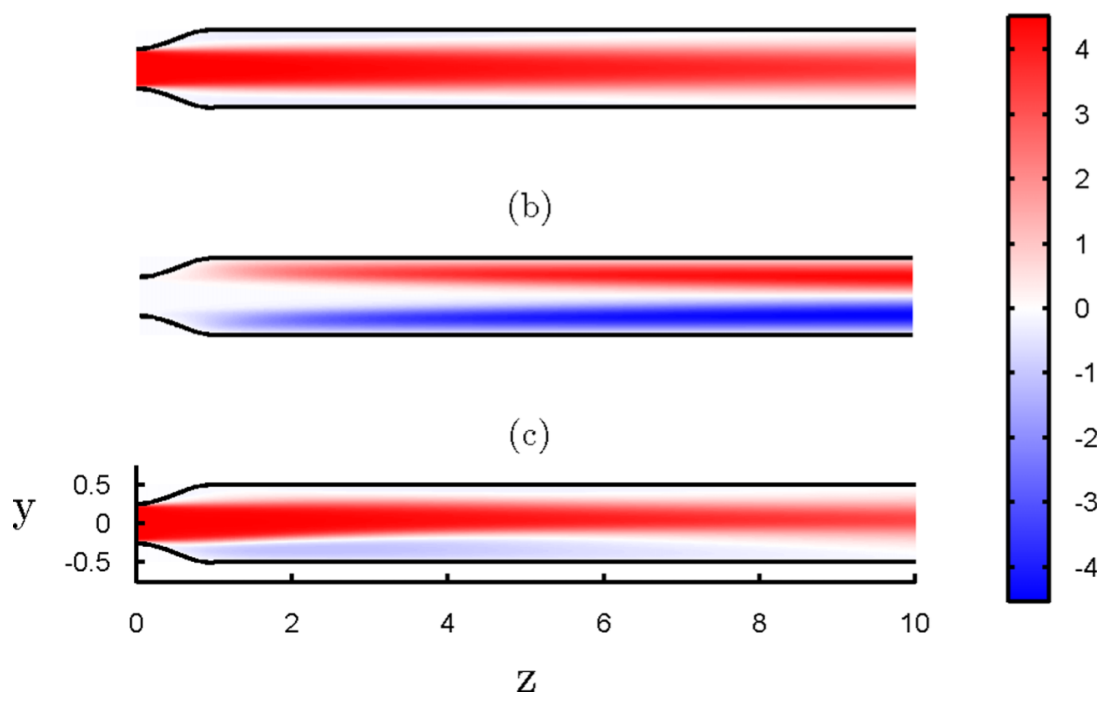

FIG. 5. Streamwise velocity in the plane $x=0$ for $R e=400, E=0$ : (a) $u_{z}$ in branch 1 , (b) leading eigenmode $U_{z}$, (c) $u_{z}$ in branch 2 . 


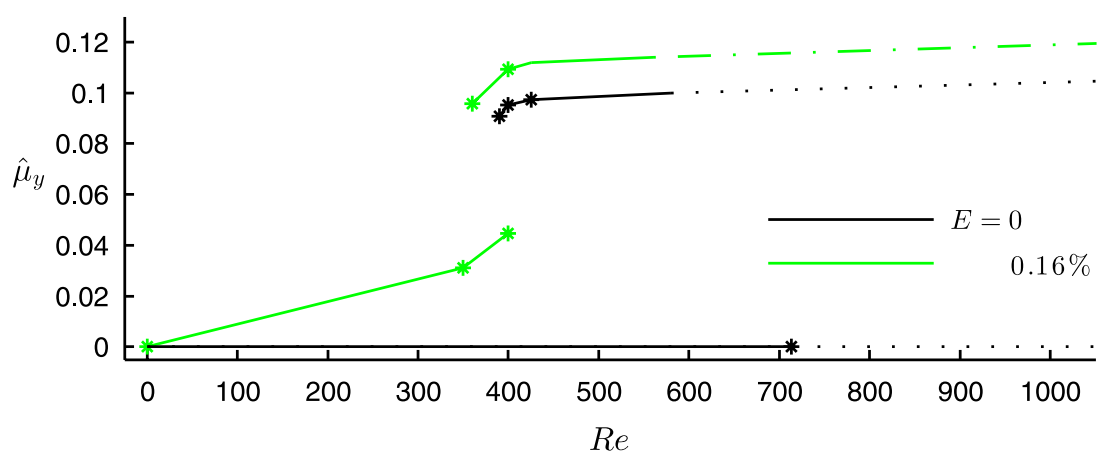

FIG. 6. Flow asymmetry as a function of Reynolds number for $E=0$ and $E=0.16 \%$. The points come from DNS data. The lines are illustrative. Solid lines show the stable, steady flow. The dotted line shows the regime of sustained intermittency and the dashed-dotted line shows the regime of splashing.

considerably more with eccentricity than that of branch 2 . Because of the difference in shape sensitivity between the two branches, the bistable region shrinks with increasing eccentricity and eventually leads to a supercritical region without hysteresis, starting at around $(E, R e)=(0.32 \%, 330)$. Because the flow of branch 1 is also more sensitive to shape variations than branch 2, the two branches converge at around $(E, R e)=(0.4 \%, 320)$, and for $E>0.4 \%$, no discontinuity between branch 1 and 2 could be observed. A preliminary investigation shows that if the jet of branch 2 is skewed towards the negative $y$-direction, then it is destabilized by an eccentricity in the positive $y$-direction. When the jet is skewed in the negative $y$-direction and an eccentricity of $E=0.16 \%$ is introduced, the flow converges to branch 1 when $R e<440$, as compared to $R e<390$ for $E=0$.

\section{F. Flow in branch 2}

The saturation process of the transition from branch 1 into branch 2 exhibits an overshoot and a settling time that grows with Reynolds number, the instability responsible for bifurcation being highly nonlinear. For $R e \geq 417$, sporadic shear layer oscillations coupled with vortex shedding occur when the amplitude of the mode reaches a local maximum. Figure 7 depicts the transition from branch 1 into branch 2 for $E=0, R e=500$ by showing the time trace of the cross-stream velocity component $u_{y}$, representing the amplitude of the unstable mode, during the settling phase into branch 2. No oscillations, however, could be observed in the flow's asymptotic state. Vortex shedding occurs for all azimuthal angles, but is more prominent where the recirculation region is thicker, which is in the lower end of the pipe, at $\phi=\pi$. Because the vortices are only shed when the extent of the recirculation region is at a temporal local extreme as the flow settles in to branch 2 , the oscillations are not continuous but intermittent. As the vortices are being convected downstream, they increase in amplitude, demonstrating a convective instability. Both Griffith et al. ${ }^{8}$ and Blackburn, Sherwin, and Barkley ${ }^{18}$ demonstrated numerically the presence of convective instabilities in axisymmetric stenotic flows (branch 1). It was also observed experimentally by Griffith et al. ${ }^{8}$ The experimental Strouhal number, St, reported by Griffith et al. ${ }^{8}$ lays within the interval $2.5<S t<5$ for $350<R e<800$. The Strouhal number in this study was found to be $2<S t<3$, depending on eccentricity and the Reynolds number. Ahmed and Giddens ${ }^{6}$ also observed shear layer oscillations in the trailing edge of the recirculation zone at $R e=500$.

The shear layer oscillations that occur during the settling phase into branch 2 , depicted in Figure 7 at $R e=500$, increase in amplitude as the Reynolds number increases. When the Reynolds number is high enough, the shear layer oscillations cause a wavy jet pattern in the region $12<$ $z<17$, followed by turbulence in $z>17$. Because the shear layer oscillations are intermittent, the wavy-turbulent pattern is temporal, giving the flow a burst-like time trace. The temporal and spatial extension of the wavy-turbulent region that emerge in $12<z<17$ increases progressively with Reynolds number. When the Reynolds number is sufficiently high $(R e \gtrsim 525)$, the wavy-turbulent region progresses upstream, up to $z \approx 4$. The turbulent region of fluid is then convected downstream 

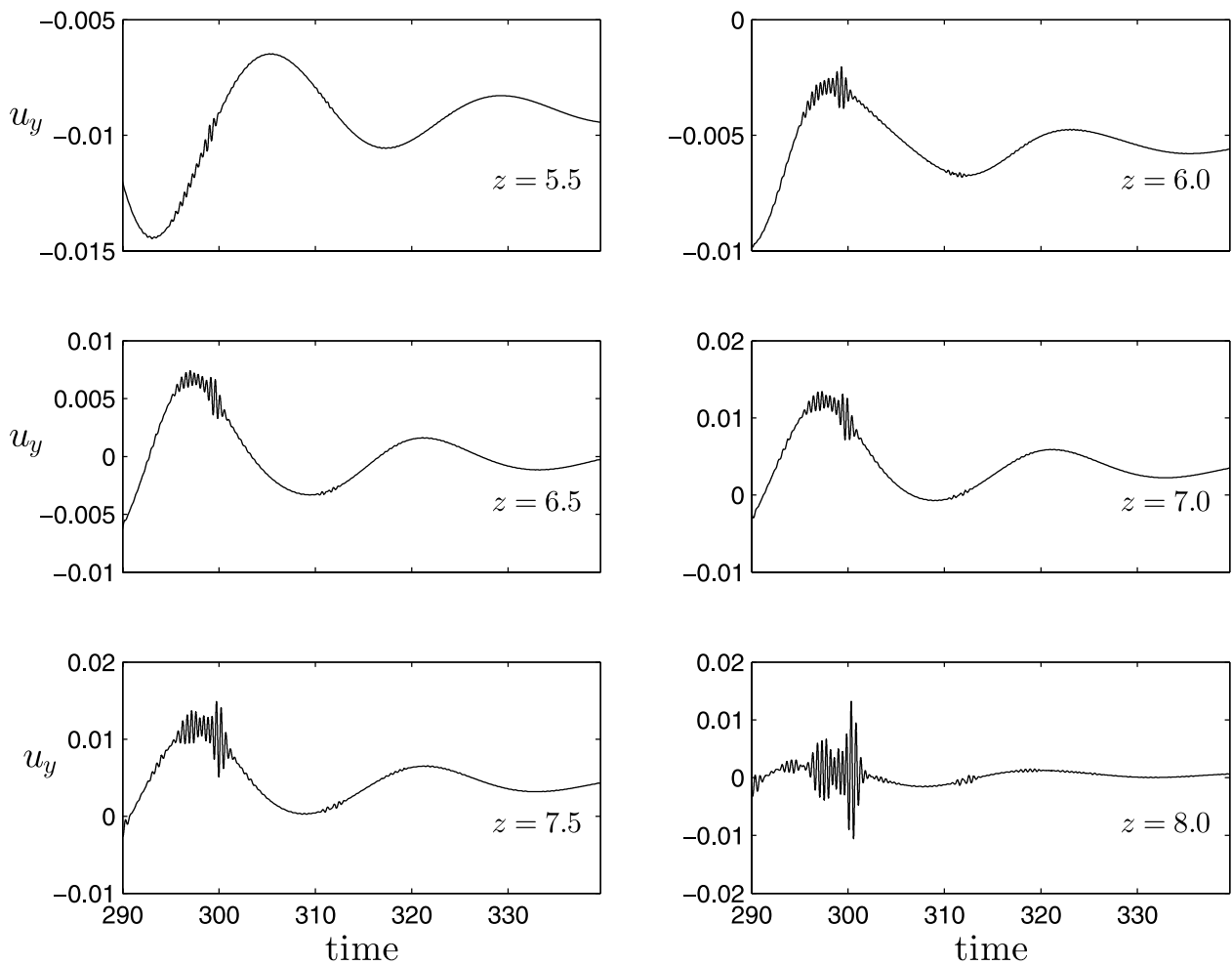

FIG. 7. Cross-stream velocity, $u_{y}$, as a function of time for six axial positions, $z$, at $R e=500$ and $E=0$. This displays the latter stages of the transition from branch 1 to branch 2 .

while the flow relaminarizes upstream. This process is depicted in Figure 8 which shows the evolution of streamwise velocity $u_{z}$ in the plane $x=0$ at different times throughout the cycle. When the flow returns to a laminar state, it is first attracted to branch 1 in the form of a symmetric, wide jet. It is then repelled to branch 2 by the growth of the leading eigenmode in branch 1 , which is why the lower recirculation region grows in thickness between $t=0$ and $t=20 \Delta t$ (Figure 8). The vortex shedding that occurs while the flow settles in to branch 2 causes the wavy-turbulent pattern to reemerge in the region $12<z<17$ at $\Delta t=18$. The wavy-turbulent flow then travels upstream and reaches $z=4$ at $\Delta t \approx 25$. The turbulence is then convected downstream and the flow relaminarizes. This pattern repeats itself with a time period of approximately $\sim 30 \Delta t$. For $575 \gtrsim \operatorname{Re} \gtrsim 525$, this intermittency pattern is transient in time, the flow settles into branch 2 after typically two or three intermittency sequences. As the Reynolds number increases further, the critical region $12<z<17$ maintains the wavy-turbulent flow without relaminarizing. For $R e \geq 560$ and $E=0.16 \%$, the flow splashes, the jet sticking to the opposite pipe wall, i.e., in negative $y$-direction, after the initial intermittency sequences have passed. For $R e \geq 580$ and $E=0$, the wavy-turbulent region progresses upstream to $z \approx 4$, after which the flow relaminarizes upstream, following the same intermittency sequence as that illustrated in Figure 8. By this mechanism, the intermittency pattern is no longer transient but sustained. The observed intermittency agrees well with experimental results by Vétel et al..$^{7}$ (Figure 8). This phenomenon was also observed by Sherwin and Blackburn. ${ }^{9}$ The intermittency pattern was largely the same as in the present study, and the long time scale fluctuations were reported to repeat themselves with a time interval of $\sim 35 \Delta t$, as compared to $\sim 30 \Delta t$ in the present study. The turbulent breakdown was reported to occur at $z \approx 10$ and progressed upstream to $z \approx 4$. Further, Sherwin and Blackburn ${ }^{9}$ also observed a flapping of the jet to the opposite pipe wall. A similar intermittency phenomenon was also seen by Lashgari et al. ${ }^{19}$ for a planar X-junction geometry. As the Reynolds number increases further, the turbulent phases become longer at the expense of laminar phases. 

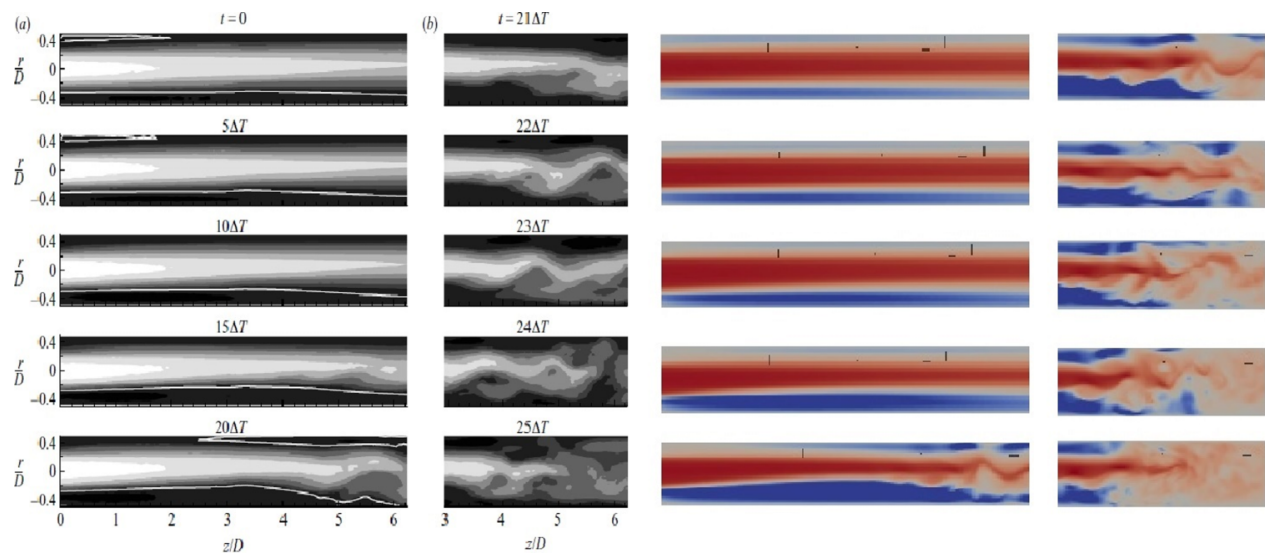

FIG. 8. Instantaneous streamwise velocity contours. Left: experimental results from Vétel et al. ${ }^{7}$ for $R e=440, \Delta t=0.94$. Right: numerical results from the present study for $R e=700$ and $\Delta t=1$.

In branch 1, the critical Reynolds number for transition to unsteady flow is $R e_{c}=713$. When a small asymmetry is introduced, however, the flow transitions to branch 2 , where the Reynolds number for transition to unsteady flow is $R e \approx 525$ and transient oscillations $R e \approx 417$. Breaking axisymmetry in stenotic flow lowers the Reynolds number for transition into unsteady flow via this mechanism.

\section{G. Discussion}

Griffith et al. ${ }^{8}$ showed via experiments that Kelvin-Helmholtz waves are formed in the shear layer between the jet and the recirculation zone. These grow as they convect downstream and cause local turbulence at $z=12$. Griffith et al. ${ }^{20}$ showed that this convective instability can be thought of as global non-modal transient growth of perturbations. ${ }^{21}$ This was also shown by Blackburn, Sherwin, and Barkley ${ }^{18}$ for a sinusoidal stenosis model. Even though these numerical investigations were limited to axisymmetric flows (branch 1), our results indicate the presence of convective instabilities in non-axisymmetric flows as well (branch 2). This is manifested by the oscillations that grow in amplitude while being transported downstream from the recirculation region, eventually causing local turbulence in $12<z<17$ and intermittency for $R e>525$. Experimental results from Griffith et al. ${ }^{8}$ indicated oscillations to begin by $R e=400$, which also is in agreement with the present study that found oscillations for $R e \geq 417$ in branch 2 .

The bifurcation diagram presented in Figure 6 is different from the one found by Sherwin and Blackburn. ${ }^{9}$ In that study, branch 2 extended down to $R e=580$ for $E=0$ instead of $R e=390$ which was found in the present study. In Sherwin and Blackburn, ${ }^{9}$ branch 2 was tracked by lowering the Reynolds number from $R e>R e_{c}=722$. By using the same method as Sherwin and Blackburn 9 and progressively lowering the Reynolds number while in branch 2 in the DNS for $E=0$ down from $R e=750$, the solution converged to branch 2 while $R e>580$. When $R e<580$, the solution converged to branch 1, thus recovering the results found by Sherwin and Blackburn. ${ }^{9}$ Hence, this discrepancy can be attributed to the different initial conditions set in the DNS. The present study was using a 3D model and found the lower threshold value of branch 2 by varying eccentricity instead of the Reynolds number, thus recovering a larger part of branch 2 than can be seen by only varying the Reynolds number for $E=0$.

The flow described by Varghese, Frankel, and Fischer ${ }^{11}$ at $E=0$ for $R e=500$ and 1000 fits the description of branch 1 . The flow was reported to be laminar and steady in both cases with a wide symmetric jet. Branch 1, however, becomes unstable at a critical Reynolds number of 713. A possible explanation is that Varghese, Frankel, and Fischer ${ }^{11}$ did not give the simulation sufficient time to be repelled from what should be an unstable solution. Supporting this explanation is the fact that an iterative method was used, slowly increasing $R e$ with a linear step increment of 10 , 

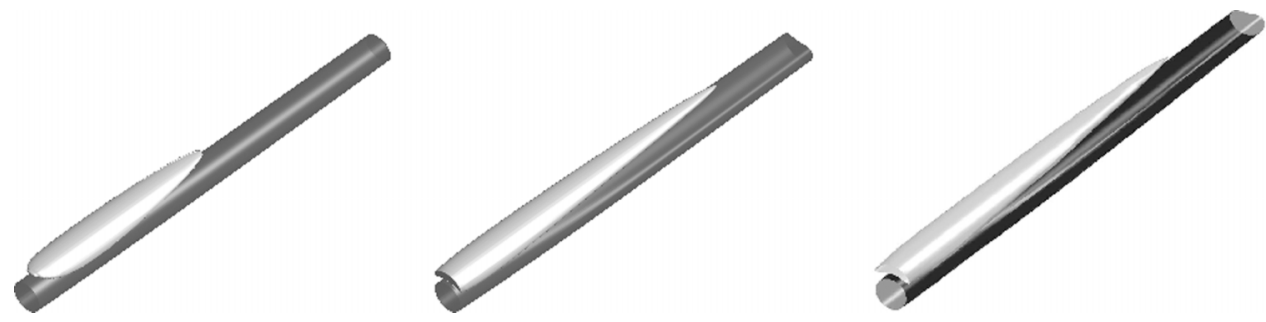

FIG. 9. $R e=350, E=0.26 \%$. Isosurfaces of the streamwise velocity for branch 1 (left image), branch 2 (middle image), and experimental results by Vétel et al. ${ }^{7}$ (right image) for the domain $1<z<8$. The dark surface represents $u_{z}=2$ and the bright $u_{z}=-0.4$. This shows that the experimental flow state reported by Vétel et al. ${ }^{7}$ belongs to branch 2 .

which should mitigate disturbances and therefore keep the solution close to that of branch 1 . For $E=5 \%$ and $R e=500$, a deflected jet of the same kind as the flow in branch 2 was found. The flow was reported to be steady, which also agrees with the asymptotic results of the present study for these parameters. For $E=5 \%$ and $R e=1000$, turbulence in the post-stenotic region for $z>4$ was reported, which also agrees with the present study's result for branch 2 .

The experimental results of Ahmed and Giddens ${ }^{6}$ at $E \approx 0$ showed discrete frequency oscillations for $R e=500$ at the trailing edge of the recirculation zone, in agreement with the present study's results on the settling phase of branch 2. For $R e=1000$, the oscillations erupted into random fluctuations, the flow being intensely turbulent, also in agreement with the present study's results on branch 2. No data were presented so a quantitative comparison is not possible.

Vétel et $a l .{ }^{7}$ modeled the stenosis as two intersecting circular arcs. As was noted earlier, one should not expect a quantitative agreement with this study, but a qualitative comparison is possible. Results from Vétel et al. ${ }^{7}$ showed a slightly asymmetric jet for low Reynolds numbers, as for the flow in branch 1 . At $R e=348$, that study found that the jet was strongly deflected to one side. Figure 9 shows isocontours for the streamwise velocity $u_{z}$ at $R e=350$ and $E=0.26 \%$ for branch 1 and branch 2 along with experimental results from Vétel et al. ${ }^{7}$ This clearly shows that experimental results by Vétel $e t a l{ }^{7}$ for $R e \geq 348$ are in excellent agreement with numerical results from branch 2; hence, it can be concluded that the experimental flow state reported by Vétel et al. ${ }^{7}$ belongs to branch 2. Since branch 2 exists only for $\operatorname{Re}>390$ at $E=0$, it is likely that there was an unintended shape asymmetry in the stenosis of the order of $E=0.3 \%$, despite the quoted tolerance of $25 \mu \mathrm{m}$ in the $2 \mathrm{~cm}$ diameter pipe, meaning that the eccentricity in theory should be lower than $0.125 \%$. The rest of the reported results are in excellent agreement with the present study for branch 2, although the critical Reynolds numbers are somewhat different. Vétel et al. ${ }^{7}$ noted oscillations over a critical Reynolds number of $R e \gtrsim 400$, which is in good agreement with this study, which noted oscillations when $R e \geq 417$. However, the critical Reynolds number for intermittency was reported to be $R e_{c} \approx 440$, whereas intermittency was observed for $R e \gtrsim 525$ in the present study. This discrepancy can presumably be explained by the different axial shape profiles of the stenosis. The intermittency patterns are similar, albeit for different Reynolds numbers. A difference is that the present study did not find the swirling motion reported by Vétel et al. ${ }^{7}$ The reason might be that the stenosis model used in the experiments did not have a shape asymmetry as has been modeled in this study in the form of eccentricity, but another more complicated, possibly semicircular, shape.

\section{CONCLUSION}

Direct numerical simulations and global linear stability analysis of the flow in a symmetric and slightly asymmetric stenoses have been carried out. The results show that, for sufficiently high Reynolds number, there are two groups of stable solutions to the Navier-Stokes equations at the same Reynolds number and eccentricity. Flows in the first group are axisymmetric for an axisymmetric geometry and nearly axisymmetric for the asymmetric cases. Flows in the second group are strongly skewed to one side for all geometries. For the perfectly symmetric case, this bistable region extends from a fold bifurcation at $R e=390$ to a subcritical bifurcation at $R e=713$. The subcritical 
bifurcation is extremely sensitive to shape asymmetry. For example, when an eccentricity of $0.27 \%$ of the pipe diameter is introduced, the Reynolds number at which the first group of solutions becomes unstable decreases from $R e_{c}=713$ to $R e_{c}=350$. By mapping all the previous experimental and numerical results into these two groups, we obtain excellent agreement between results for both steady and transient flow behaviors. $7,6,8,9,11,12$

The first group of solutions remains steady all the way up to the subcritical bifurcation. The second group has a thicker recirculation zone and a thinner shear layer, however, and is more susceptible to shear layer instability. For $R e \geq 417$, transient shear layer oscillations occur on the second group, which then grow in amplitude while being convected downstream. The transition number to intermittent flow in this group $(R e \approx 525)$ is much lower than that of the first group of solutions $(R e=713)$. Therefore, breaking axisymmetry in a stenotic flow lowers the Reynolds number for transition into unsteady flow by provoking non-oscillatory transition from the first group to the second group, followed by growth of oscillations in the second group. For $R e \gtrsim 525$, the oscillations grow to a sufficiently large amplitude for local turbulence to be caused downstream of the stenosis, which travels upstream until the flow is turbulent for $z>4$. This is followed by relaminarization, generating an intermittency sequence that repeats itself a few times before the flow settles in. For $R e \geq 580$ and $E=0$, the intermittency is sustained and the sequence repeats itself at a regular frequency, the flow being highly intermittent. As the Reynolds number increases further, the turbulence reaches further upstream and the turbulent phases become longer at the expense of the laminar phases.

The results presented here show that there are two branches of steady states in stenotic flows at Reynolds numbers typical of arterial flow $(R e \sim 400)$. The first branch in which the flow is closest to being symmetric, becomes unstable at much lower Reynolds numbers when the stenosis is even slightly non-axisymmetric. The flow then grows to the second branch, in which the central jet is skewed to one side. Both branches develop into an unsteady state when the Reynolds number increases, but this occurs at lower Reynolds number for the second branch than it does for the first. We suggest that these two effects explain the discrepancies between previous numerical and experimental studies because tiny asymmetries are inevitable in experiments but not in numerical studies. These asymmetries generate flows that become unsteady at lower Reynolds numbers, leading to earlier turbulent transition and intermittency than that predicted numerically. Most importantly, arterial stenoses are always asymmetric and are therefore likely to be more turbulent than numerical studies suggest. Future studies should be conducted to quantify the effect of asymmetry on the hydrodynamic stability of stenotic flows under more realistic arterial conditions, e.g., with a pulsatile inlet profile and non-Newtonian flow models.

\section{ACKNOWLEDGMENTS}

This work was supported by the European Research Council through Project No. ALORS 259620. Computer time provided by the University of Cambridge is gratefully acknowledged. The authors would also like to thank Dr. Jérôme Vetel for providing experimental data from Ref. 7 for comparison (Figures 8 and 9).

${ }^{1}$ G. Burch, "The etiology of arteriosclerosis-A thought," Am. Heart J. 83, 434-436 (1972).

${ }^{2}$ C. Weber and H. Noels, "Artherosclerosis: Current pathogenesis and therapeutic options," Nat. Med. 11, 1410-1422 (2011).

${ }^{3}$ R. W. Hobson, D. G. Weiss, W. S. Fields, J. Goldstone, W. S. Moore, J. B. Towne, and B. W. Creighton, "Efficacy of carotid endarterectomy for asymptomatic carotid stenosis," N. Engl. J. Med. 328, 221-227 (1993).

${ }^{4}$ V. Peiffer, S. J. Sherwin, and P. D. Weinberg, "Does low and oscillatory wall shear stress correlate spatially with early atherosclerosis? A systematic review," Cardiovasc. Res. 99, 242-250 (2013).

${ }^{5}$ D. N. Ku, D. P. Giddens, C. K. Zarins, and S. Glagov, "Pulsatile flow and atherosclerosis in the human carotid bifurcation. Positive correlation between plaque location and low oscillating shear stress," Arterioscler., Thromb., Vasc. Biol. 5, 293-302 (1985).

${ }^{6}$ S. A. Ahmed and D. P. Giddens, "Velocity measurements in steady flow through axisymmetric stenoses at moderate Reynolds numbers," J. Biomech. 16, 505-516 (1983).

${ }^{7}$ J. Vétel, A. Garon, D. Pelletier, and M.-I. Farinas, "Asymmetry and transition to turbulence in a smooth axisymmetric constriction,” J. Fluid Mech. 607, 351-386 (2008).

${ }^{8}$ M. D. Griffith, T. Leweke, M. C. Thompson, and K. Hourigan, "Steady inlet flow in stenotic geometries: Convective and absolute instabilities," J. Fluid Mech. 616, 111-133 (2008). 
${ }^{9}$ S. J. Sherwin and H. M. Blackburn, "Three-dimensional instabilities and transition of steady and pulsatile axisymmetric stenotic flows," J. Fluid Mech. 533, 297-327 (2005).

${ }^{10}$ T. Panitz and D. T. Wasan, "Flow attachment to solid surfaces: The Coanda effect," AIChE J. 18, 51-57 (2004).

${ }^{11}$ S. S. Varghese, S. H. Frankel, and P. F. Fischer, "Direct numerical simulation of stenotic flows. Part 1. Steady flow," J. Fluid Mech. 582, 253-280 (2007).

${ }^{12}$ M. D. Griffith, T. Leweke, M. C. Thompson, and K. Hourigan, "Effect of small asymmetries on axisymmetric stenotic flow," J. Fluid Mech. 721, R1-1-R1-11 (2013).

${ }^{13}$ B. K. Bharadvaj, R. F. Mabon, and D. P. Giddens, "Steady flow in a model of the human carotid bifurcation. Part I-Flow visualization," J. Biomech. 15(5), 349-362 (1982).

${ }^{14}$ P. F. Fischer, J. W. Lottes, and S. G. Kerkemeier, nek5000 web page, 2008, http://nek5000.mcs.anl.gov.

${ }^{15}$ K. J. Maschhoff and D. C. Sorensen, "P_ARPACK: An efficient portable large scale eigenvalue package for distributed memory parallel architectures," in Proceedings of the Third International Workshop on Applied Parallel Computing, Industrial Computation and Optimization, PARA '96 (Springer-Verlag, London, UK, 1996), pp. 478-486.

${ }^{16}$ O. Tammisola, F. Giannetti, V. Citro, and M. P. Juniper, "Second-order perturbation of global modes and implications for spanwise wavy actuation,” J. Fluid Mech. 577, 314-335 (2014).

${ }^{17}$ H. Baumgärtel, Perturbation Theory for Matrices and Operators (Akademie Verlag, Berlin, 1984).

${ }^{18}$ H. M. Blackburn, S. J. Sherwin, and D. Barkley, "Convective instability and transient growth in steady and pulsatile stenotic flows," J. Fluid Mech. 607, 267-277 (2008).

${ }^{19}$ I. Lashgari, O. Tammisola, V. Citro, and M. P. Juniper, “The planar x-junction flow: Stability analysis and control," J. Fluid Mech. 753, 1-28 (2014).

${ }^{20}$ M. D. Griffith, M. C. Thompson, T. Leweke, and K. Hourigan, "Convective instability in steady stenotic flow: Optimal transient growth and experimental observation," J. Fluid Mech. 655, 504-514 (2010).

${ }^{21}$ P. J. Schmid, “Nonmodal stability theory,” Annu. Rev. Fluid Mech. 39, 129-162 (2007). 